\title{
SOLVING MULTI-OBJECTIVE PROBLEM ON SUPPLY CHAIN PERFORMANCE MEASURE BY MULTI- OBJECTIVE EVOLUTIONARY ALGORITHM
}

\begin{tabular}{|l|l|}
\hline Susmita Bandyopadhyay & Indraneel Mandal \\
Department of Business Administration & School of Management Studies \\
The University of Burdwan, Burdwan-713104 & IMS Unison University \\
West Bengal, India & Dehradun-248001, Uttarakhand, India \\
Email: bandyopadhyaysusmita2010@gmail.com & Email: indraneel75@gmail.com \\
\hline
\end{tabular}

\begin{abstract}
In this paper, we have proposed a new multiobjective supply chain problem related to the performance measure of a supply chain. The problem consists of four objectives, viz., 1) minimization of total cost, 2) minimization of total inventory, 3) minimization of bullwhip effect, and 4) maximization of service level. These four objectives are the expressions for the four performance measuring factors for a supply chain. The problem has been solved by two algorithms 1) modified Nondominated Sorting Genetic Algorithm-II (NSGAII) and 2) the original NSGA-II. Comparison between the experimental results for the two algorithms shows that the proposed modified NSGA-II performs significantly better than the original NSGA-II.
\end{abstract}

Keywords- Multi-Objective Evolutionary Algorithm, Nondominated Sorting Genetic Algorithm-II (NSGA-II), Supply Chain, Performance Measure

\section{INTRODUCTION}

A supply chain is an integrated view of interconnected business organizations (known as echelons) that are connected to each other through some form of business transactions. The echelons of a serial supply chain may consist of supplier, manufacturer, distributor, wholesaler, retailer (Figure 1). The benefits of these business organizations in a modern supply chain are inter-linked to one another which results in the need to consider these echelons in an integrated fashion. Thus maximizing the benefits or minimizing the losses or expenses of an entire supply chain is a very important issue to consider.

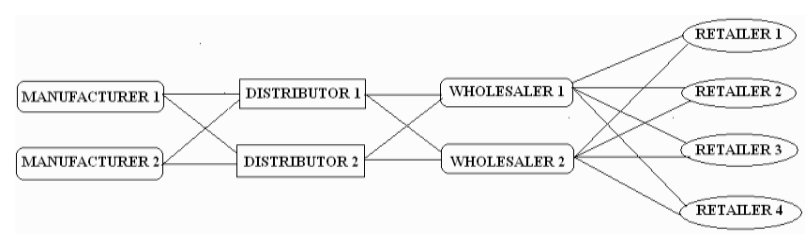

Fig. 1. A Serial Supply Chain

In this regard, this paper deals with the supply chain performance. The performance of a supply chain can be measured by various factors. The most important ones among these factors are: 1) the total cost of a supply chain, 2) the total inventory of a supply chain, 3 ) the bullwhip effect of a supply chain, 4) the service level. The total cost may consist of the transportation costs, the inventory holding costs, the shortage costs and so on. The total inventory represents the aggregate inventory of all the echelons of a supply chain. The bullwhip effect is "the amplification in the fluctuation of order, as we move upwards in a supply chain from the retailer to the manufacturer" (Bhattacharya and Bandyopadhyay [1]). Service level measures the extent to which the customers have been serviced. There are different types of service level concepts. In this paper, a service level in terms of 'the fulfillment of customer demands from the inventory' has been considered.

\section{LITERATURE REVIEW}

A multi-objective optimization problem consists of more than one objective along with the constraints. However a multi-objective optimization problem may be solved either by mathematical approach or by various nature based algorithms such as Genetic Algorithm, Differential Evolution, Particle Swarm Optimization, Honey Bee Mating Algorithm, Simulated Annealing, and hybrid algorithms.

Most of the research papers on supply chain dealing with multi-objective problems are on supplier selection problem, such as research work by Ozkok et al. [2] and designing and planning problems, such as research work by Giarola et al. [3]. Among some other areas of research on the application of multi-objective approach on supply chain include the research studies of Deshpande et al. [4], Langella and Zanoni [5]. Among the various multi-objective algorithms proposed in the literature, the benchmark ones are - Non-dominated Sorting Genetic Algorithm (NSGA-II) [6], Strength Pareto Evolutionary Algorithm (SPEA2) [7], Pareto Archived Evolutionary Strategy (PAES) [8], Pareto Envelop-based Selection Algorithm (PESA-II) [9], Niched Pareto Genetic Algorithm (NPGA-II) [10]. Some of other significant research studies include the studies of Jaimes et al. [11], Jun-jie et al. [12]. In spite of the presence of a number of original algorithms, hybrid algorithms and modified algorithms, the literature still lacks a superior multi-objective evolutionary algorithm that can solve problems with more than two objectives. In this paper, we have modified the original NSGA-II to deal with the multi-objective problem proposed in this paper.

\section{PROBLEM FORMULATION}

Before presenting the formulation of the problem, the notations used and the assumptions taken are presented below. The assumptions, notations and the formulated problem are shown in Figure 2, Figure 3 and Figure 4 respectively. 


\section{ELK}

\section{Asia Pacific Journals}

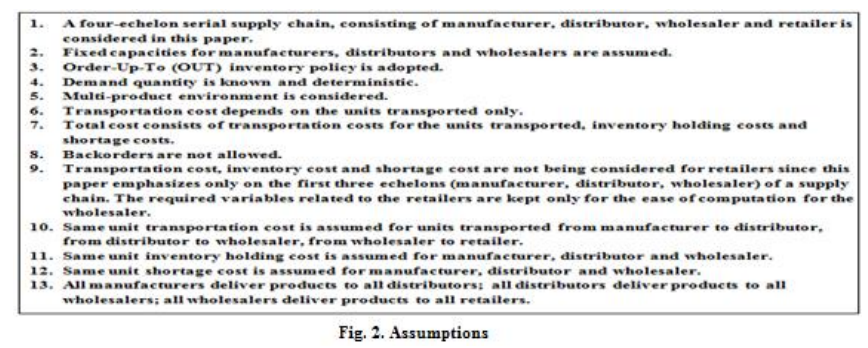

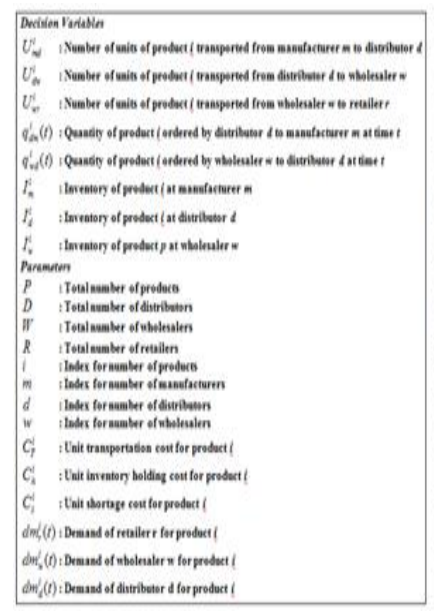

Fig, 3, Notations

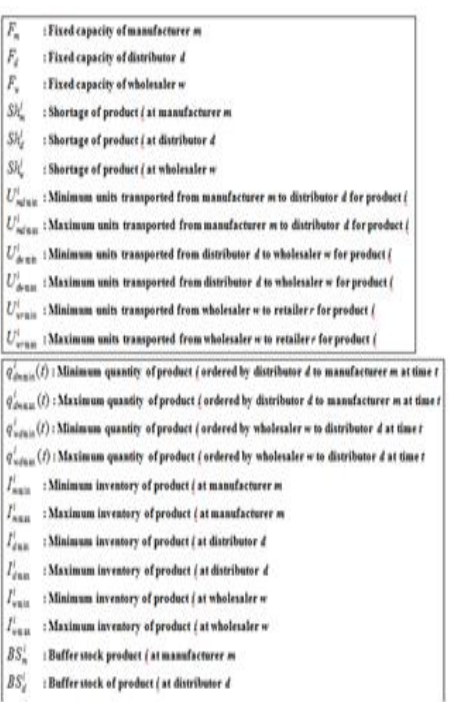

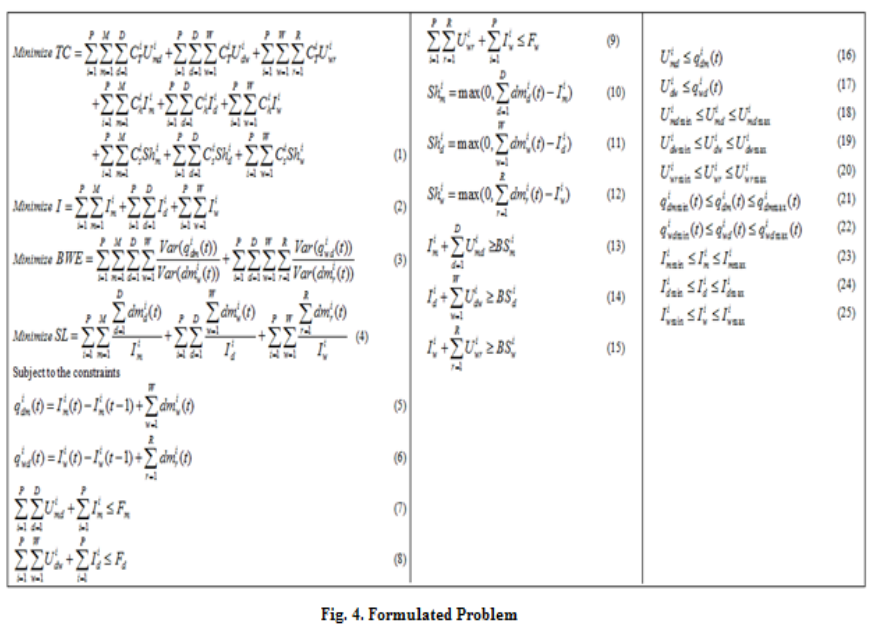

Objective (1) minimizes the total cost of a supply chain. The first three terms of (1) represent the transportation costs for the units transported from manufacturers to distributors, distributors to wholesalers and wholesalers to retailers respectively. The next three terms represent the inventory holding costs at the manufacturers, the distributors and the wholesalers respectively. The last three terms represent the inventory shortage costs at the manufacturers, the distributors and the wholesalers respectively. Objective (2) represents the total inventory at the manufacturers (first term), the distributors (second term) and the wholesalers (last term). Objective (3) represents the total bullwhip effect (BWE) which is a ratio of the order variability to the demand variability. The first and second terms of (3) represent the BWE as faced by the distributors and the wholesalers respectively. Objective (4) represents the service level which is defined as the ratio of the total demand and total inventory.

Constraints (5) and (6) are the expressions for the order quantities for the OUT inventory policy. Constraint (5) says that the quantity ordered for product $i$ by distributor $d$ to manufacturer $m$ is the sum of the difference between the inventories at the current and previous periods at the distributor $d$ and the total demand of all the wholesalers for that particular distributor. Likewise constraint (6) is the expression for order quantity by wholesaler to distributor. Constraints (7), (8) and (9) are the constraints for fixed capacities of the manufacturers, the distributors and the wholesalers. Constraints (10), (11) and (12) are the expressions for the shortages of product $i$. Constraint (13) means that the sum of the total inventory of product $i$ at the manufacturer $m$ and the total units transported from manufacturer $m$ to all the distributors is greater than the buffer stock at the manufacturer $m$. Constraint (14) means that the sum of the total inventory of product $i$ at the distributor $d$ and the total units transported from the distributor to all the wholesalers is greater than the buffer stock at the distributor $d$. Constraint (15) means that the sum of the total inventory of product $i$ at the wholesaler and the units transported from the wholesaler $w$ to all the retailers greater than the buffer stock at wholesaler. Constraints (16) and (17) ensure that the units transported for a product should be less than the quantity ordered. Constraints (18) to (25) define the upper and lower limits of the decision variables.

\section{PROPOSED ALGORITHM}

The algorithm proposed in this paper is shown in Figure 5. The main components of the proposed algorithm are discussed below.

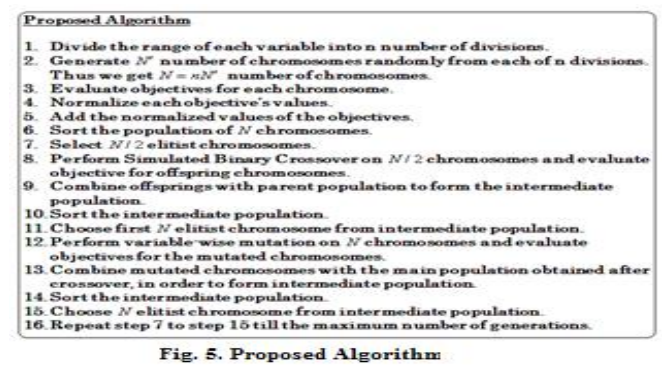

\section{A. Initialization}

The decision variables' spaces are divided into n number of divisions and an equal number of chromosomes are chosen randomly from each of the divisions in order to maintain a good spread of chromosomes over the variables' spaces. Next, we have normalized each objective by expression (26). Then the normalized values have been added. The result can be thought of as fitness for the chromosomes. These values can be used easily to select the chromosomes. Here, $V$ is the value of an objective for a chromosome, $V$ min is the minimum value of that objective in the population, $V \max$ is the maximum value of that objective in the population. In this way the algorithm will be able to solve problems with two more objectives. The genotype of the chromosomes as used in this paper is given in Figure 7. 


\section{, \\ ELK \\ Asia Pacific Journals}

Normalized Value $=\frac{V-V_{\min }}{V_{\max }-V_{\min }}$

\section{B. Crossover and Mutation}

The probabilities of both the crossover and the mutation are 1 , which means that both the crossover and the mutation are certain events in the proposed algorithm. The purpose of making mutation, a certain event, is to introduce more diversity in the population. The algorithm for mutation is depicted in Figure 6. The mutation algorithm takes the same approach as the initialization - dividing the population into $n$ parts and distributing the chromosomes into each part equally.

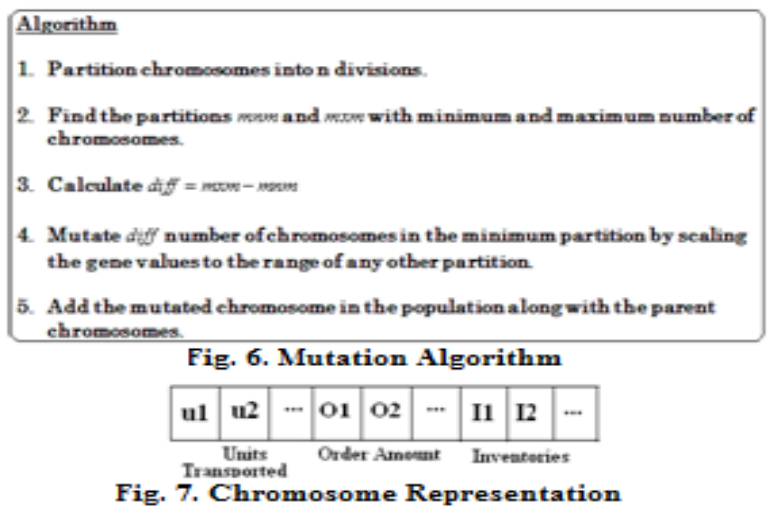

The experimentation has been conducted in Matlab software in a PC with $2.8 \mathrm{GHz}$ and $2 \mathrm{~GB}$ memory. Both the proposed algorithm and NSGA-II [6] have been used to solve the proposed problem. The population size has been taken to be 100 and the number of generations has been varied from 10 to 100 with an interval of 10 , that is, the number of generations are $10,20,30, \ldots, 100$. The probabilities of crossover and mutation are both 1 for the proposed algorithm. Since for the proposed algorithm, the crossover and mutation are certain events, thus we had to choose a fixed probability value for the original NSGA-II, which will show the best results. For the original NSGA-II, a number of experiments have been performed with probability values of $0.1,0.2$, $0.3, \ldots, 0.9$ and the crossover probability is set to 0.9 since the probability value of 0.9 provides the best results for the original NSGA-II.

Table 1 shows the average values of all the objectives for both the proposed algorithm and NSGA-II. The average values for the proposed algorithm are observed to be significantly better than those for NSGA-II for objective 1, objective 2 and objective 3 . For objective 4 , both algorithms fail to perform well since no value for objective 4 is less than or equal to or around 1. For the first 4 values, the proposed algorithm performs worse than NSGA-II and for the next five values, the proposed algorithm performs better. Thus as a whole, both algorithms perform nearly same for the average values of objective 4 . Table 2 shows the minimum values of all the objectives for both the proposed algorithm and NSGA-II.
ELK Asia Pacific Journals - Special Issue ISBN: 978-81-930411-4-7
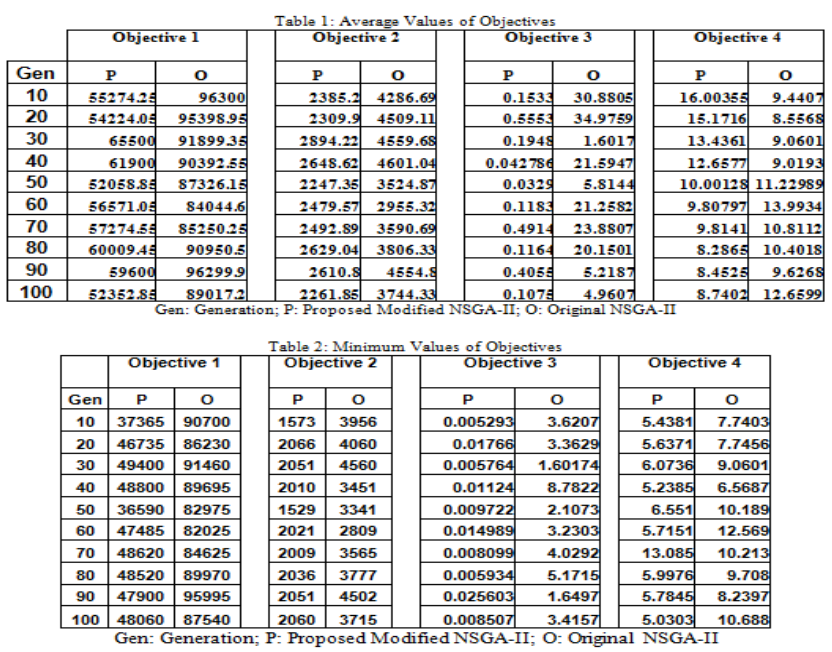

\begin{tabular}{|c|c|c|c|c|c|c|c|}
\hline \multicolumn{4}{|c|}{ Proposed Algorithm } & \multicolumn{4}{|c|}{ NSGA-II } \\
\hline Objective 1 & Objective 2 & Objective 3 & Objective 4 & Objective 1 & Objective 2 & Objective 3 & Objective 4 \\
\hline 49295 & 2141 & 0.011311 & 8.961 & 105000 & 3979 & 8.8002 & 10.064 \\
\hline 48265 & 2116 & 0.031628 & 7.785 & 92450 & 4142 & 3.8776 & 9.6921 \\
\hline 48200 & 2111 & 0.032559 & 6.796 & 91025 & 4133 & 16.154 & 9.4141 \\
\hline 48055 & 2143 & 0.014841 & 7.126 & 91680 & 4067 & 4.321 & 10.041 \\
\hline 48185 & 2108 & 0.035108 & 8.832 & 92445 & 4140 & 3.8776 & 9.6952 \\
\hline 48315 & 2117 & 0.035493 & 8.768 & 91860 & 4132 & 3.9363 & 9.7501 \\
\hline 48100 & 2137 & 0.008642 & 7.17 & 90955 & 4090 & 16.022 & 9.6692 \\
\hline 48000 & 2140 & 0.018005 & 6.158 & 91070 & 4097 & 15.953 & 9.6671 \\
\hline 48185 & 2143 & 0.014841 & 7.131 & 105000 & 3978 & 92.653 & 10.179 \\
\hline 61450 & 2582 & 0.037372 & 7.649 & 95135 & 4692 & 5.6938 & 8.3628 \\
\hline
\end{tabular}

The Pareto optimal solutions for proposed algorithm show significantly better result than those of NSGA-II. A fraction of the solutions is shown in Table 3 above. Table 3 shows the first 10 Pareto optimal solutions for both proposed algorithm and NSGA-II at generation 10. The reason for using such low generation number is to show that, even at such an early generation, the proposed algorithm performs better than NSGA-II. Since this paper deals with a multi-objective problem, thus the true comparison of the results can be performed only through Pareto optimal solutions. Figure 8 shows the Pareto optimal solutions for the proposed modified NSGA-II and the original NSGA-II. Figures $8 \mathrm{a}, 8 \mathrm{~b}, 8 \mathrm{c}$ and $8 \mathrm{~d}$ show the Pareto optimal solutions for the generation numbers $70,80,90$ and 100 respectively. These figures show the 4dimensional scatter plot drawn by dplot software. The meaning of the representative legends are mentioned only in Figure 8a. Figure 8 clearly shows that the modified NSGA-II performs significantly better than the original NSGA-II for the all the generations except for generation 80 which shows better results for the original NSGA-II. From the above results and discussions it is clear that the proposed algorithm solves the proposed supply chain problem remarkably better than NSGA-II. 


\section{ELK}

Asia Pacific Journals

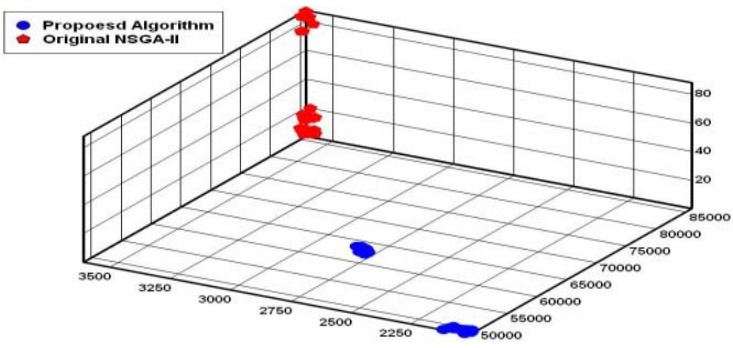

(a)

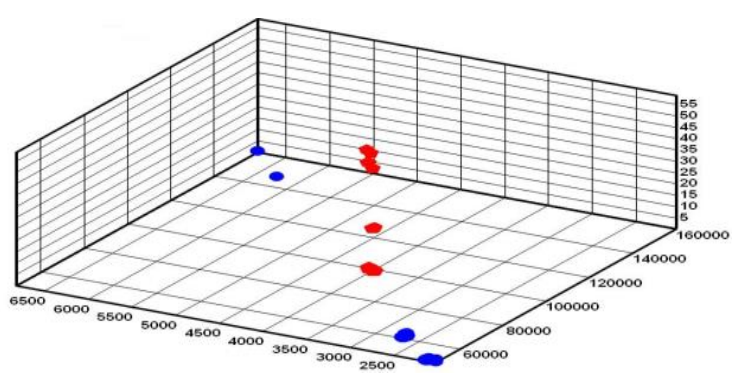

(b)

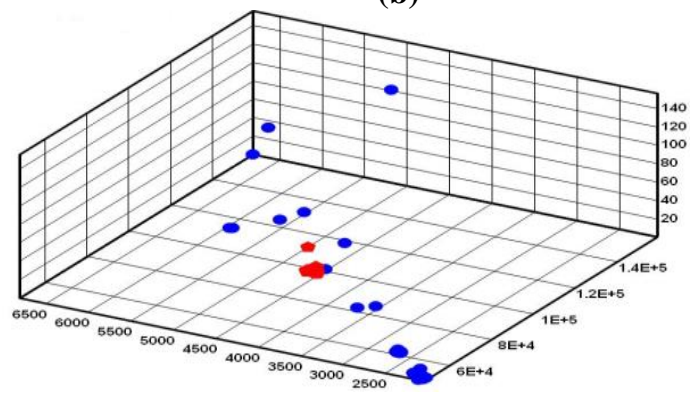

(c)

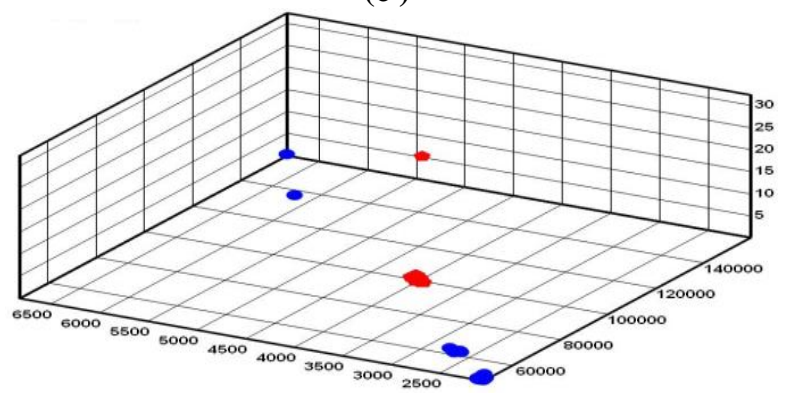

(d)

Fig. 8. Pareto Optimal Solutions

\section{References}

[1] R. Bhattacharya, and S. Bandyopadhyay, "A review of the causes of bullwhip effect in a supply chain", International Journal of Advanced Manufacturing Technology, vol. 54(912), pp. 1245-1261, 2011.

[2] A. Ozkok Beyza, F. Tiryaki, "A compensatory fuzzy approach to multi-objective linear supplier selection problem with multiple-item", Expert Systems with Applications, vol. 38, pp. 11363-11368, 2011.

[3] S. Giarola, A. Zamboni, and F. Bezzo, "Supply Chain Design and Capacity Planning: from First to Second
Generation Biofuel Systems", Chemical Engineering Transactions, vol. 24, pp. 253-258, 2011.

[4] P. Deshpande, D. Shukla, and M. K. Tiwari, "Fuzzy goal programming for inventory management: A bacterial foraging approach", European Journal of Operational Research, vol. 212(2), pp. 325-336, 2011.

[5] M. Langella Ian, and S. Zanoni, "Eco-efficiency in logistics: a case study on distribution network design", International Journal of Sustainable Engineering, vol. 4(2), pp. 115-226, 2011.

[6] K. Deb, A. Pratap, S. Agarwal, and T. Meyaruvan, "A Fast and Elitist Multiobjective Genetic Algorithm: NSGA-II", IEEE Transactions on Evolutionary Computation, vol. 6(2), pp. 182-197, 2002.

[7] E. Zitzler, M. Laumanns, and L. Thiele, "SPEA2: Improving the Strength Pareto Evolutionary Algorithm". In K. Giannakoglou, D. Tsahalis, J. Periaux, P. Papailou, and T. Fogarty (Eds.), EUROGEN 2001. Evolutionary Methods for Design, Optimization and Control with Applications to Industrial Problems, pages 95-100, Athens, Greece, 2001.

[8] D. Knowles Joshua, and D. W. Corne, "Approximating the nondominated front using the Pareto Archived Evolution Strategy", Evolutionary Computation, vol. 7(3), pp. 1-26, 1999.

[9] D. W. Corne, N. R. Jerram, J. D. Knowles, and M. J. Oates, "PESA-II: Regionbased Selection in Evolutionary Multiobjective Optimization". In L. Spector, E. D. Goodman, A.Wu, W. Langdon, H.-M. Voigt, M. Gen, S. Sen, M. Dorigo, S. Pezeshk, M. H. Garzon, and E. Burke (Ed.). Proceedings of the Geneticand Evolutionary Computation Conference (GECCO’2001), pages 283-290, San Francisco, California. Morgan Kaufmann Publishers, 2001.

[10] M. Erickson, A. Mayer, and J. Horn, "The Niched Pareto Genetic Algorithm 2 Applied to the Design of Groundwater Remediation Systems". In E. Zitzler, K. Deb, L. Thiele, C. A. Coello Coello, and D. Corne (Ed.). First International Conference on Evolutionary Multi-Criterion Optimization, pages 681-695. Springer-Verlag. Lecture Notes in Computer Science No. 1993, 2001.

[11] A. L. Jaimes, A. Coello Carlos Coello, H. Aguirre, and K. Tanaka, "Adaptive Objective Space Partitioning Using Conflict Information for Many-Objective Optimization". Lecture Notes in Computer Science (including subseries Lecture Notes in Artificial Intelligence and Lecture Notes in Bioinformatics) 6576 LNCS, pp. 151-165, 2011.

[12] Y. Jun-jie, Z. Jian-zhong, F. Ren-cun, L. Xiao-hai, and L. Li, "Multi-objectives particle swarm optimization based on adaptive grid algorithms", Journal of System Simulation, vol. 22(21), pp. 5843-5847, 2008. 